BMJ Open Diabetes Research \& Care

\title{
Low basal metabolic rate as a risk factor for development of insulin resistance and type 2 diabetes
}

Sebastian Maciak (D) , ${ }^{1}$ Diana Sawicka, ${ }^{2}$ Anna Sadowska, ${ }^{2}$ Sławomir Prokopiuk, ${ }^{2,3}$ Sylwia Buczyńska, ${ }^{1}$ Marek Bartoszewicz, ${ }^{1}$ Gabriela Niklińska, ${ }^{4}$ Marek Konarzewski, ${ }^{1}$ Halina Car $^{2}$

To cite: Maciak S, Sawicka D, Sadowska A, et al. Low basal metabolic rate as a risk factor for development of insulin resistance and type 2 diabetes. BMJ Open Diab Res Care 2020;8:e01381. doi:10.1136/ bmjdrc-2020-001381

Received 19 March 2020 Revised 1 June 2020 Accepted 8 June 2020
Check for updates

(C) Author(s) (or their employer(s)) 2020. Re-use permitted under CC BY-NC. No commercial re-use. See rights and permissions. Published by BMJ.

${ }^{1}$ Faculty of Biology, University of Bialystok, Bialystok, Poland ${ }^{2}$ Faculty of Health Sciences, Medical University of Bialystok, Bialystok, Poland

${ }^{3}$ Faculty of Health Sciences, Lomza State University of Applied Sciences, Lomza, Poland

${ }^{4}$ Faculty of Veterinary Medicine, Warsaw University of Life Sciences, Warsaw, Poland

Correspondence to Dr Sebastian Maciak; maciaks@uwb.edu.pl

\section{ABSTRACT}

Introduction Identification of physiological factors influencing susceptibility to insulin resistance and type 2 diabetes (T2D) remains an important challenge for biology and medicine. Numerous studies reported energy expenditures as one of those components directly linked to T2D, with noticeable increase of basal metabolic rate (BMR) associated with the progression of insulin resistance. Conversely, the putative link between genetic, rather than phenotypic, determination of BMR and predisposition to development of T2D remains little studied. In particular, low BMR may constitute a considerable risk factor predisposing to development of T2D.

Research design and methods We analyzed the development of insulin resistance and T2D in 20-week-old male laboratory mice originating from three independent genetic line types. Two of those lines were subjected to divergent, non-replicated selection towards high or low body mass-corrected BMR. The third line type was nonselected and consisted of randomly bred animals serving as an outgroup (reference) to the selected line types. To induce insulin resistance, mice were fed for 8 weeks with a high fat diet; the T2D was induced by injection with a single dose of streptozotocin and further promotion with high fat diet. As markers for insulin resistance and T2D advancement, we followed the changes in body mass, fasting blood glucose, insulin level, lipid profile and $M T O R$ expression.

Results We found BMR-associated differentiation in standard diabetic indexes between studied metabolic lines. In particular, mice with low BMR were characterized by faster body mass gain, blood glucose gain and deterioration in lipid profile. In contrast, high BMR mice were characterized by markedly higher expression of the $m T O R$, which may be associated with much slower development of T2D.

Conclusions Our study suggests that genetically determined low BMR makeup involves metabolism-specific pathways increasing the risk of development of insulin resistance and T2D.

\section{INTRODUCTION}

Diabetes mellitus is a chronic metabolic disease, which has become a leading health concern worldwide. ${ }^{1-3}$ The two types of diabetes are type 1 diabetes and type two

\section{Significance of this study}

What is already known about this subject?

- Type 2 diabetes (T2D) development is underlined by both genetic and environmental factors affecting energy balance and leading to a nexus of weight gain and insulin resistance.

- Interpretation of observational study results is usually confounded by the lack of clear discrimination of genetic and environmental factors, as well as their overall correlative character.

What are the new findings?

- Individuals with genetically determined low basal metabolic rate (BMR) have higher propensity to spontaneous weight gain and insulin resistance, while their high BMR counterparts manifested much slower development of T2D.

- Increase in liver mTOR expression fosters a transition toward catabolic processes enabling the maintenance of high metabolism rate, acting as a protection shield against disturbances in glucose homeostasis.

\section{How might these results change the focus of} research or clinical practice?

- Incorporation of BMR-related approach in studies of metabolic diseases, including diabetes, should be of significant importance to indicate patients with predisposition to insulin resistance and T2D.

diabetes (T2D), with much higher prevalence of T2D, which increases general multimorbidity posing social and economic burden. ${ }^{4}$

T2D development is underlined by both genetic and environmental factors affecting energy balance and leading to a nexus of weight gain and insulin resistance (IR) ${ }^{5-8}$ In humans, no less than $70 \%$ of the total energy expenditures constitutes basal metabolic rate (BMR) or resting metabolic rate (RMR), which comprises of energy expended for vital functions in a resting, postabsorptive state. ${ }^{9}$ It is therefore unsurprising that the link between variation in $\mathrm{BMR} / \mathrm{RMR}$ and weight 
gain has received a lot of attention. ${ }^{5} 710-13$ However, the results of observational studies were largely inconclusive. For example, several authors concluded that individuals characterized by lower energy expenditures and sedentary lifestyle may be at higher risk of weight gain and impaired insulin sensitivity. ${ }^{5}{ }^{14-16}$ Conversely, there is even evidence for positive association between RMR, weight gain and/or diabetes of both types, ${ }^{141718}$ whereas Katzmarzyk et $a l^{19}$ and Morino $e t a l^{20}$ found no association between RMR and changes in body mass, fatness, or diabetes prevalence.

Interpretation of observational study results is usually confounded by the lack of clear discrimination of genetic and environmental factors, as well as their overall correlative character. Well-established research paradigms addressing such inconsistencies include the use of animal models tailored to pinpoint physiological and molecular mechanisms driving the observed correlations. To date, various animal models of diabetes have been developed. ${ }^{132122}$ Yet, to our knowledge, no animal model has ever been applied to study the putative link between genetically determined variation in $\mathrm{BMR} / \mathrm{RMR}$ and predisposition to T2D development. Here, we analyzed this link using lines of laboratory mice divergently selected for high and low BMR, currently characterized by a $50 \%$ differentiation in BMR that is unmatched by any other animal model. ${ }^{1623}$ Apart from the difference in BMR, the lines differ distinctly with respect to the relative sizes of metabolically active internal organs, ${ }^{23}$ as well as metabolic risk indicators, such as percent of body fat mass, blood lipid profile, and fasting blood glucose levels. ${ }^{16}$ Thus, both physiological and anatomical properties of our selection lines make them a suitable model for testing the effect of genetically determined BMR on susceptibility of metabolic disorders, including T2D.

At the genetic and molecular level, the most important intracellular pathway that coordinates both glucose and insulin homeostasis is the mechanistic target of the rapamycin (mTOR) signaling pathway. mTOR is a conserved serine/threonine kinase belonging to the PI3K-related kinase family, which senses and integrates a variety of metabolic and nutrient cues to regulate basic biological processes such as cell metabolism, growth, and survival. $^{224-26}$ An increasing number of studies indicate that mTOR activity is involved in development of metabolic disorders, including obesity, and T2D. ${ }^{2} 24252728$ However, the effect of mTOR on progression of IR and T2D seems ambiguous. ${ }^{28}{ }^{29}$ Under physiological conditions, the high concentration of cellular nutrients (especially glucose and proteins) leads to the activation of the $m T O R$ gene in metabolically active organs like liver or muscles, contributing to cellular glucose intake and protecting against hyperglycemia. ${ }^{2} 242627$ Numerous studies also reported the positive effect of the mTOR protein on the regulation of insulin excretion, $\beta$-cells survival, and proliferation. ${ }^{25} 27$ Conversely, hyperactivation of mTOR may lead to the degradation of membrane insulin receptors, disrupting glucose transportation and promoting IR. ${ }^{225}$

In the present study, we examined the putative link between genetic determination of BMR and predisposition to development of T2D using lines of mice introduced above. In particular, we analyzed the between-line difference in expression of the mTOR gene following T2D progression. As markers for IR and T2D advancement, we followed the changes in concentration of fasting blood glucose, insulin level, and lipid profile in two experimental setups, that is, mice fed with high fat diet alone or with a streptozotocin (STZ)-promoted high-fat diet (HFD), emulating IR and T2D development, respectively. Thus, using this specific animal model, we directly tested the significance of genetically determined metabolic variation as a risk factor of susceptibility to develop IR and T2D, which may help to clarify inconsistencies of longitudinal cohort studies on humans.

\section{RESEARCH DESIGN AND METHODS}

\section{Animals and experimental setup}

We analyzed the development of IR and T2D in 20-week-old male laboratory mice (Mus musculus) originating from three independent genetic line types. Two of those lines were subjected to divergent, non-replicated artificial selection towards high (H-) or low (L-) body mass-corrected BMR (for more details see ref 30). The third line type was non-selected (NS) and consisted of randomly bred animals serving as an outgroup (reference) to the selected line types. ${ }^{30}$ We used 156 mice, where 120 originated from the 53rd generation of a longterm selection (60 from each BMR line type) and 36 mice from the 18th generation of the NS line type.

Following measurements of BMR, animals in each line type were randomly assigned to one of the experimental group: IR, T2D, and standard control (20 per group from each BMR line type and 12 per group from the NS line type). To induce IR, mice from the IR group were fed for 8 weeks with an HFD (chow no. D12492, Labofeed, Poland) with $60 \%$ fat content. ${ }^{31}$ For the T2D group, our objective was to elicit the state of severe hyperglycemia, as reported in our previous studies (eg, see ref 32). In a classic study models (eg, refs 3,22 ), this is achieved by means of the exposure to the HFD followed by STZ treatment. However, in comparison with the L-BMR line, the H-BMR mice are much more resistant to development of prediabetic condition elicited by the HFD. ${ }^{16}$ To equalize the initial conditions, we therefore reversed the sequence of treatments and subject the T2D mice to STZ followed by 8 weeks of HFD. We used a single dose of STZ $(100 \mathrm{mg}$ / kg; Sigma-Aldrich, St Louis, Missouri, USA) selected in a pilot study carried out with doses of STZ of 80, 100, and $120 \mathrm{mg} / \mathrm{kg}$. The control group received the standard murine chow containing $7 \%$ fat (Labofeed, Poland).

To measure fasting blood glucose level, the tail vein of all animals was punctured with a sterile needle every 2 weeks, and a blood droplet was used to perform blood glucose 
test (Diagomat, Diagnosis, Poland). Additionally, hyperglycemia was checked (blood glucose over $130 \mathrm{mg} / \mathrm{dL}$ ) in T2D group 72 hours after STZ injection. In the control group, the fasting blood glucose levels were measured at the beginning and at the end of the experiment only.

Throughout the experiment, animals were maintained individually in plastic cages at $23^{\circ} \mathrm{C}$ and $12 \mathrm{~d}: 12 \mathrm{n}$ photoperiod. Body mass was recorded every 2 weeks. At the end of the 8th week, they were anesthetized by intraperitoneal administration of a mixture of ketamine and xylazine at a dose of $50 \mathrm{mg} / \mathrm{kg}$ and $5 \mathrm{mg} / \mathrm{kg}$, respectively. The whole blood was taken directly from the heart, centrifuged immediately, and serum was collected. A piece of liver $(\sim 50 \mathrm{mg})$ was taken and suspended in RNA stabilization solution in order to be used for genetic analyses (RNA later, Invitrogen, Lithuania). The serum and liver were stored at $-80^{\circ} \mathrm{C}$ until analyzed.

\section{Metabolic rate measurements}

Following standard procedure, BMR was measured in all animals, before the experiment. ${ }^{30}$ Briefly, we used a positive-pressure open-circuit respirometry system with dried and warmed atmospheric air pushed through the system. The system sequentially monitored oxygen consumption rate of mice placed individually in three $350 \mathrm{~cm}^{3}$ chambers. The chambers were submerged in a water bath set at $32^{\circ} \mathrm{C} \pm 0.1^{\circ} \mathrm{C}$ (thermoneutral zone for mice). The airstream from the chambers was directed to a computer-controlled channel multiplexer, a part of a Sable Systems TR-1 oxygen analyzer (Henderson, Nevada, USA). The air was sampled at a rate of $75 \mathrm{~mL} /$ min, and prior to passing through an oxygen sensor (S-3A/I Applied Electrochemistry, Pittsburgh, PA, USA), it was scrubbed off $\mathrm{CO}_{2}$ (Carboabsorb AS, BDH Laboratory Supplies, Lutterworth, UK) and moisture (Drierite, Drierite Co. LTD, Xenia, Ohio, USA). Each metabolic measurement trial lasted 3 hours, and oxygen concentrations in each chamber were recorded every second for 2 hours. Animals were fasted for 6 hours before BMR measurements. We defined BMR $(\mathrm{mL}$ $\left.\mathrm{O}_{2} / \mathrm{h} \pm 0.0013\right)$ as the lowest rate of oxygen consumption that did not change for at least $4 \mathrm{~min}$ by more than $0.01 \%$. Metabolic data were analyzed with a Sable System DATACAN V software.

\section{Biochemical parameters}

The plasma insulin level was determined with an ELISA (Mercodia AB, Uppsala, Sweden). High-density lipoprotein (HDL) and low-density lipoprotein (LDL) cholesterol fractions and triglycerides (TGs) blood concentration were measured with the BioAssay Systems (HDL/LDL assay kit E2H:-100 and TG assay kit ETGA200, respectively). Homeostasis model assessmentinsulin resistance (HOMA-IR) was used to assess $\beta$-cell function and IR from basal glucose and insulin according to the formula: fasting glucose $(\mathrm{mmol} / \mathrm{L}) \times$ fasting insulin $(\mu \mathrm{U} / \mathrm{mL}) / 22.5 .{ }^{3}$

\section{mTOR expression}

The total tissue RNA was isolated from the liver sample with a use of standard commercial kit (Total RNA mini plus, A\&A Biotechnology, Poland). DNAse I treatment with Clean-up RNA concentrator (A\&A Biotechnology, Poland) was applied to remove traces of DNA from all samples. RNA purity was checked using the NanoDrop 2000 (ThermoFisher). Primer specific, high capacity cDNA reverse transcription kit was used to convert RNA to cDNA (TranScriba 1 step PCR Mix, A\&A Biotechnology, Poland). All RNA samples were adjusted to a starting concentration of $50 \mathrm{ng} / \mu \mathrm{L}$. The expression levels of $m T O R$ and $\beta$-actin genes (as a reference) were estimated with SYBR Green on real-time PCR (Applied Biosystems). $\Delta$ Ct values for mTOR in experimental groups were corrected for untreated controls

Table 1 Mean values for basal metabolic rate and body mass on the beginning (ST) and the end (END) of experiment (least square means \pm SE) in H-BMR, L-BMR, and NS-BMR line type mice originated from insulin resistant (IR), type 2 diabetes (T2D), and control group

\begin{tabular}{|c|c|c|c|c|}
\hline Treatment & Line type & BMR (mL $\left.\mathrm{O}_{2} / \mathrm{h}\right)$ & Body mass ST (g) & Body mass END (g) \\
\hline \multirow[t]{3}{*}{ IR } & H-BMR & $64.54 \pm 1.28^{\mathrm{a}}$ & $34.2 \pm 0.64^{\mathrm{a}}$ & $34.8 \pm 0.82^{\mathrm{a}, \mathrm{ns}}$ \\
\hline & L-BMR & $39.18 \pm 0.97^{b}$ & $33.4 \pm 0.93^{\mathrm{a}}$ & $40.9 \pm 1.02^{\mathrm{b}, \star \star \star}$ \\
\hline & NS-BMR & $51.86 \pm 0.98^{c}$ & $32.5 \pm 0.87^{\mathrm{a}}$ & $34.1 \pm 1.27^{\mathrm{a}, \mathrm{ns}}$ \\
\hline \multirow[t]{3}{*}{ T2D } & H-BMR & $63.22 \pm 1.17^{\mathrm{a}}$ & $33.17 \pm 0.41^{a}$ & $31.1 \pm 1.74^{\mathrm{a}, \mathrm{ns}}$ \\
\hline & L-BMR & $41.02 \pm 0.68^{b}$ & $35.5 \pm 0.76^{a}$ & $29.5 \pm 3.08^{a, *}$ \\
\hline & NS-BMR & $52.95 \pm 0.74^{c}$ & $33.9 \pm 0.91^{\mathrm{a}}$ & $28.4 \pm 1.65^{a, \star \star}$ \\
\hline \multirow[t]{3}{*}{ Control } & H-BMR & $63.07 \pm 1.24^{\mathrm{a}}$ & $33.3 \pm 0.88^{\mathrm{a}}$ & $33.3 \pm 0.87^{\mathrm{a}}$ \\
\hline & L-BMR & $40.78 \pm 0.93^{b}$ & $35.2 \pm 0.87^{a}$ & $35.4 \pm 0.82^{\mathrm{a}}$ \\
\hline & NS-BMR & $53.06 \pm 0.87^{\mathrm{c}}$ & $33.0 \pm 0.61^{\mathrm{a}}$ & $34.6 \pm 0.67^{a}$ \\
\hline
\end{tabular}

Letters $(a, b, c)$ show significant differences between line types within the control, IR and T2D groups. Asterisks indicate statistical significance of the difference between the respective experimental groups and their controls within each line type $\left({ }^{\star} p<0.05,{ }^{* *} p<0.01,{ }^{* * *} p<0.001\right)$. BMR, basal metabolic rate; H-BMR, high basal metabolic rate; L-BMR, low basal metabolic rate; ns, not significant; NS-BMR, non-selected basal metabolic rate. 


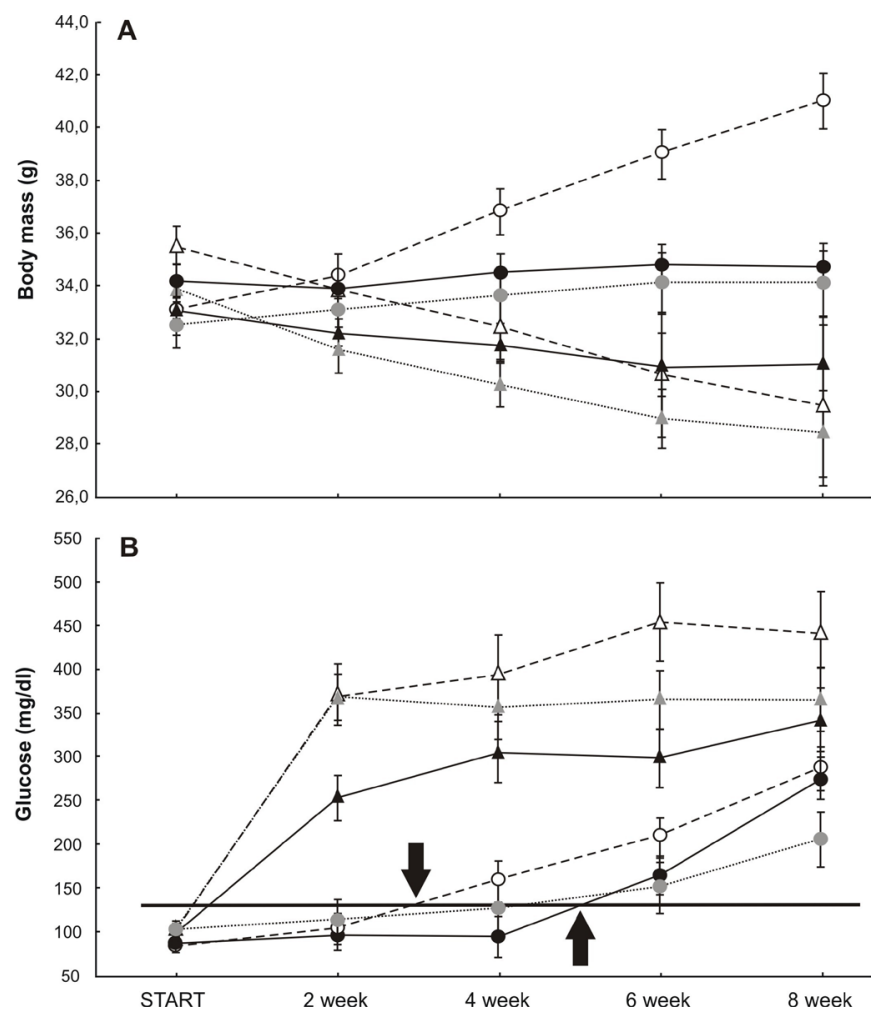

Figure 1 Body mass (A) and fasting blood glucose levels (B) during the experiment in $\mathrm{H}$-BMR (solid lines), L-BMR (dashed lines), and NS-BMR (spotted lines) mice originated from insulin resistant (circles) and type 2 diabetes (triangles) group. Arrows indicate the average blood glucose level above $130 \mathrm{mg} / \mathrm{dL}$ (insulin resistance) for L-BMR (third week) and H-BMR (fifth week). Data are expressed as least square means. Solid horizontal line indicates hyperglycemic (ie, $130 \mathrm{mg} / \mathrm{dL}$ ) state for Swiss Webster laboratory mice. H-BMR, high basal metabolic rate; L-BMR, low basal metabolic rate; NS-BMR, non-selected basal metabolic rate.

with average $\Delta \mathrm{Ct}$ as the calibrator, according to $2^{-\Delta \Delta \mathrm{Ct}}$ method. ${ }^{33}$

\section{Statistical analyses}

BMR was analyzed by means of analysis of covariance with the line type and experimental group as fixed factors and body mass as a covariate. The differences in the body mass and blood parameters were analyzed with two-way analysis of variance, with the line type and experimental group as fixed factors. The changes in blood glucose during the experiment were analyzed with ANOVA for repeated measurements with line type and experimental group as fixed factors. Post hoc comparisons were performed with a Fisher's LSD test. All statistical analyses were carried out with Statistica V.13.3 software (StatSoft, Poland). P $<0.05$ was considered statistically significant.

\section{Data and resource availlability}

The data sets generated and/or analyzed during the current study are available from the corresponding author on request.
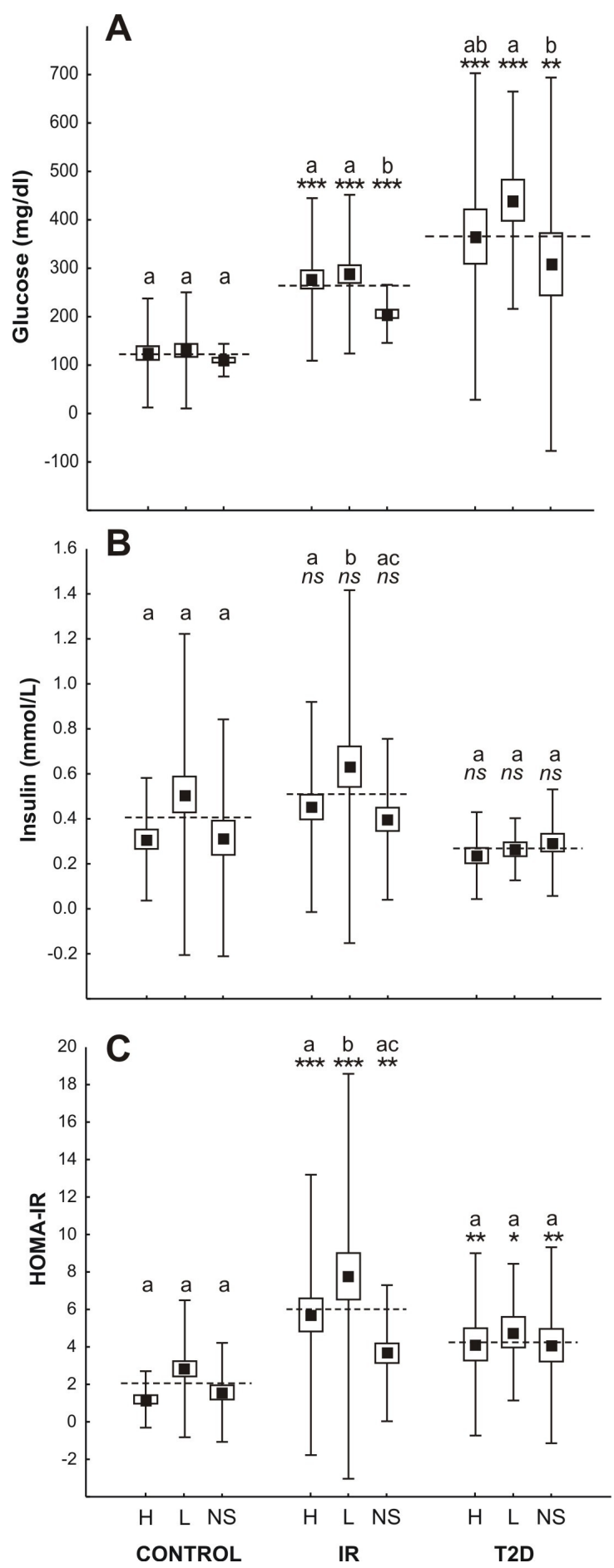

Figure 2 Final fasting blood glucose levels (A), insulin blood level (B), and HOMA-IR index (C) in three different metabolic lines (H-, L-, NS-; high, low, non-selected basal metabolic rate, respectively) of mice originated from insulin resistant (IR), type two diabetes (T2D), and control group. Data are expressed as least square means (solid boxes) \pm SE (open boxes) with \pm doubledSD (whiskers). Dashed lines depict means in each experimental group. a,b,c show significant differences between line types within the control, IR and T2D groups. Asterisks indicate statistical significance of the difference between the respective experimental groups and their controls within each line type $\left({ }^{*} p<0.05,{ }^{* *} p<0.01\right.$, ${ }^{* * *} \mathrm{p}<0.001$; ns, not significant). HOMA-IR, homeostasis model assessment-insulin resistance. 


\section{RESULTS}

\section{BMRs and body mass}

Mice originating from each specific line type differed significantly with respect to the metabolic rate $\left(\mathrm{F}_{2,148}=768.71 ; \mathrm{p}<0.0001\right)$. The average $\mathrm{BMR}$ values in high line type were $63.7 \pm 0.71 \mathrm{~mL} \mathrm{O}_{2} /$ hour, while individuals from the L-BMR line were characterized with values $60 \%$ lower, that is, $40.3 \pm 0.50 \mathrm{~mL} \mathrm{O}_{2} /$ hour (table 1). Animals from the NS line type had intermediate values for this trait $(52.6 \pm 0.50 \mathrm{~mL} \mathrm{O}$ /hour; table 1).

Initial body mass did not differ between line types $\left(\mathrm{F}_{2,147}=2.62 ; \mathrm{p}=0.076\right)$ nor experimental groups $\left(\mathrm{F}_{2,147}=0.71 ; \mathrm{p}=0.493\right)$. However, body mass gain rate differed during the experiment (time $\times$ line type interaction: $\left.\mathrm{F}_{8,272}=2.15 ; \mathrm{p}<0.0001\right)$ with the L-BMR individuals gaining weight faster (figure $1 \mathrm{~A}$ ).

\section{Carbohydrate profile and IR}

Plasma glucose level increased significantly in all experimental groups (the effect of time in repeated measures ANOVA: $\left.\mathrm{F}_{4,276}=123.1, \mathrm{p}<0.0001\right)$ but also differed between the IR and T2D treatment groups $\left(\mathrm{F}_{1,69}=41.88, \mathrm{p}<0.0001\right)$ and between the line types $\left(\mathrm{F}_{2,69}=4.96, \mathrm{p}<0.01\right)$, with L-BMR mice gaining blood glucose significantly faster than other mice (time $\times$ line type interaction: $\mathrm{F}_{8,276}=5.23$; $\mathrm{p}<0.000$, figure $1 \mathrm{~B}$ ). The hyperglycemic state (fasting blood glucose over $130 \mathrm{mg} / \mathrm{dL}$ ) was achieved on third, fourth, and fifth consecutive week in L, NS, and H -BMR line respectively (figure $1 \mathrm{~B}$ ).

The final blood glucose level during the 8th week of the experiment was significantly affected by the line type and experimental group $\left(\mathrm{F}_{2,116}=10.35\right.$; $\mathrm{p}<0.0001$ and $\quad \mathrm{F}_{2,116}=60.25 ; \quad \mathrm{p}<0.0001, \quad$ respectively; figure 2A), with insignificant line type by group interaction $\left(\mathrm{F}_{4,116}=2.44 ; \mathrm{p}=0.051\right)$. Interestingly, the L-BMR glucose level was slightly higher than in other line types in both the IR and T2D groups (figure 2A). Conversely, insulin level differed between experimental groups but not between genetic lines $\left(\mathrm{F}_{2,104}=5.67 ; \mathrm{p}=0.005\right.$ and $\mathrm{F}_{2,104}=2.49 ; \mathrm{p}=0.09$, respectively). In general, animals kept on an HFD only (IR group) were characterized by the highest concentration of insulin in peripheral blood, while individuals originated from T2D group had lower level of this hormone compared with control groups (figure 2B).

The adopted experimental model led to development of IR and T2D in all groups studied. The estimation of IR expressed by HOMA-IR index was significantly affected by both, experimental group and line type $\left(\mathrm{F}_{2,105}=17.09\right.$; $\mathrm{p}<0.0001$ and $\mathrm{F}_{2,105}=3.55 ; \mathrm{p}=0.032$, respectively with HOMA-IR $>2.5$ in all experimental groups; figure 2C). Line type by group interaction was not significant $\left(\mathrm{F}_{4,105}=1.10 ; \mathrm{p}=0.362\right)$. HOMA-IR was markedly higher in low BMR animals fed with fat rich diet (figure 2C), which indicated rapid progression of IR leading to T2D in these animals.
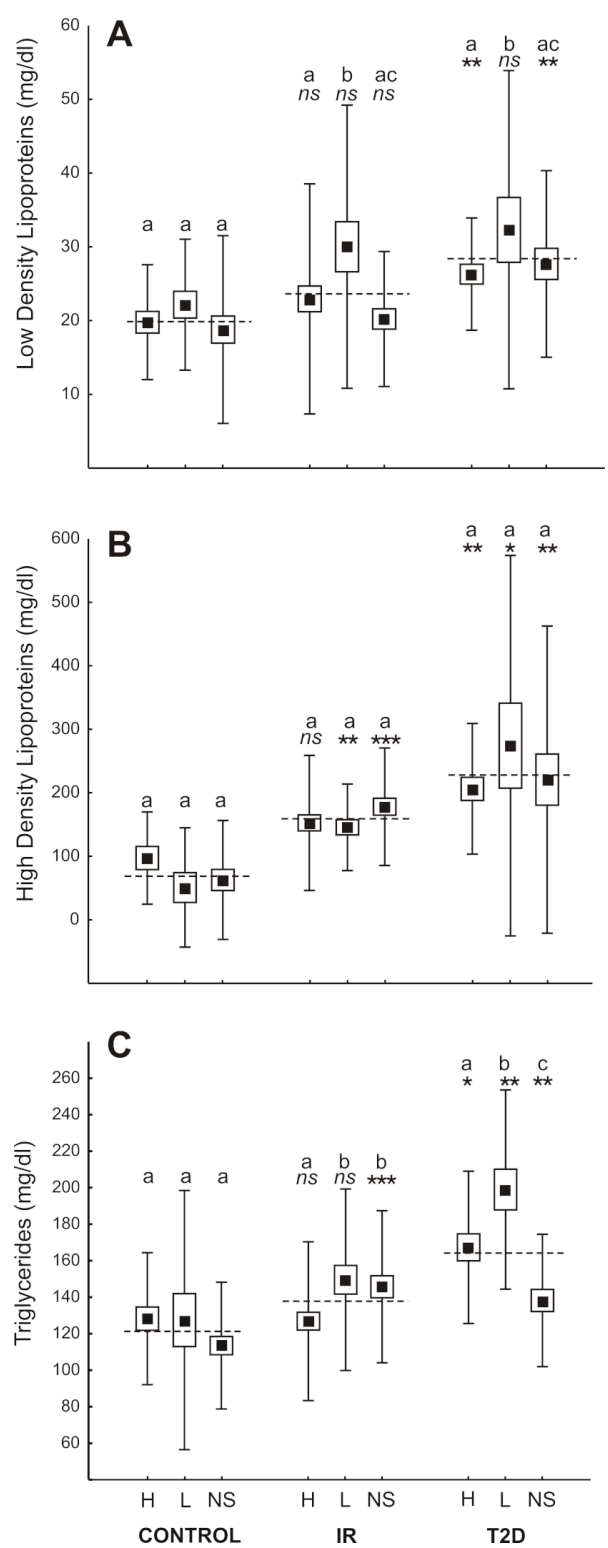

Figure 3 Lipids profile (LDL cholesterol (A), HDL cholesterol $(B)$, and triglycerides $(\mathrm{C}))$ in three different metabolic lines (H-, L-, NS-; high, low, non selected basal metabolic rate, respectively) of mice originated from insulin resistant (IR), type 2 diabetes (T2D), and control group. Data are expressed as least square means (solid boxes) $\pm S E$ (open boxes) with \pm doubled SD (whiskers). Dashed lines depict means in each experimental group. a,b,c show significant differences between line types within the control, IR and T2D groups. Asterisks indicate statistical significance of the difference between the respective experimental groups and their controls within each line type $\left({ }^{*} p<0.05,{ }^{* *} p<0.01\right.$, ${ }^{* *} p<0.001$; ns, not significant). HDL, high-density lipoprotein; LDL, lowdensity lipoprotein.

\section{Lipid profille}

Cholesterol fractions differed significantly between experimental groups $\left(\mathrm{F}_{2,67}=23.12 ; \mathrm{p}<0.0001 ; \mathrm{F}_{2,78}=8.75\right.$; $\mathrm{p}<0.001$ for HDL and LDL, respectively), with the highest values found in T2D groups (figure 3). Line type affiliation had significant impact on the concentration of low density lipoproteins $\left(\mathrm{F}_{2,78}=5.00 ; \mathrm{p}<0.01\right)$, with markedly 


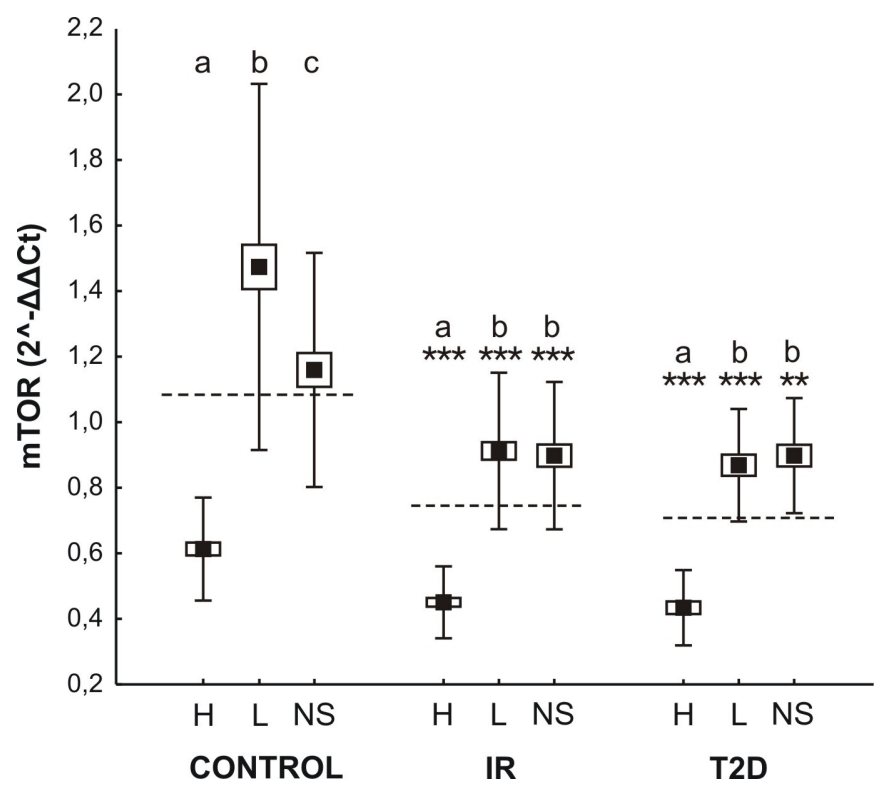

Figure 4 mTOR expression in three metabolic lines $(\mathrm{H}-$, L-, NS-; high, low, non selected basal metabolic rate, respectively) of mice originated from insulin resistant (IR), type two diabetes (T2D), and control group. Data are expressed as least square means (solid boxes) \pm SE (open boxes) with \pm doubled SD (whiskers). Dashed lines depict means in each experimental group. a,b,c show significant differences between line types within the control, IR and T2D groups. Asterisks indicate statistical significance of the difference between the respective experimental groups and their controls within each line type $\left({ }^{\star *} p<0.01,{ }^{\star \star \star} p<0.001\right)$.

higher level of LDL in LBMR individuals (figure 3A). HDL concentration remained statistically insignificant in all groups studied $\left(\mathrm{F}_{2,67}=0.02 ; \mathrm{p}=0.98\right.$; figure $\left.3 \mathrm{~B}\right)$. Line type by group interaction was insignificant for both HDL $\left(\mathrm{F}_{4,67}=1.24 ; \mathrm{p}=0.30\right)$ and LDL $\left(\mathrm{F}_{4,78}=0.65 ; \mathrm{p}=0.63\right)$. Exposure of studied animals to a high fat and STZ-promoted high fat diet led to significant increase in the amount of TGs in all line types $\left(\mathrm{F}_{2,82}=8.82 ; \mathrm{p}<0.001\right)$, with the highest concentrations of TG in the T2D groups (except for the NS-T2D group; figure 3C). Line type by treatment interaction was significant $\left(\mathrm{F}_{4,82}=3.93 ; \mathrm{p}=0.006\right)$.

\section{mTOR expression}

Both the HFD and STZ promoted HFD were characterized by an increase in the expression of mTOR in all metabolic groups $\left(\mathrm{F}_{2,107}=71.11 ; \mathrm{p}<0.0001\right.$; figure 4$)$. Between-line differences in the amount of mRNA for a specific gene were also observed $\left(\mathrm{F}_{2,107}=172.25 ; \mathrm{p}<0.0001\right)$, with markedly higher expression in groups characterized by high BMR (figure 4 ). The line type by group interaction was statistically significant $\left(\mathrm{F}_{4,107}=10.92 ; \mathrm{p}<0.0001\right)$. Presented results may suggest an enhanced mechanism of glucose turnover in organisms with genetically higher BMR which, in turn, may delay IR and T2D.

\section{DISCUSSION}

Recent genome-wide association studies have identified more than 90 loci implicated in human obesity and the propensity to develop T2D. ${ }^{8435}$ Despite this clearly polygenetic nature, the great majority of animal models of human obesity and/or diabetes are purposely monogenetic and therefore mimic only a narrow spectrum of mechanisms underlying T2D progression. ${ }^{21} 223637$ Most importantly, to our knowledge, none of the mouse models of T2D in use today have been developed with variation of BMR in mind. Since BMR constitutes an important risk factor for development of T2D in humans, the lack of consideration for BMR variation in the animal models may undermine their clinical relevance. ${ }^{11} 1338$ As both obesity ${ }^{39} 40$ and $\mathrm{BMR}^{23}$ are highly heritable, selection on traits directly related to variation in the rate of energy expenditures should provide an ample opportunity for identification of metabolic risk factors of development of T2D. Here, we demonstrated that mice with genetically determined low BMR have higher propensity to spontaneous weight gain and IR, while their high BMR counterparts manifested much slower development of T2D. Thus, the line types of mice used therein may be a useful model for clarification of the mechanisms linking BMR, the major component of human energy budgets, with T2D.

\section{BMR-related glucose changes in high fat and STZ treated mice}

Several previous studies have compared glycemic and insulinogenic responses of different strains of mice fed a high fat or regular diet. In general, an increase in fasting glucose level in most HFD models is observed after 4 weeks of treatment, with $\mathrm{C} 57 \mathrm{Bl} / 6 \mathrm{~J}$ mouse line developing a more severe diabetic phenotype than other lines. ${ }^{374142}$ In our study, LBMR mice displayed hyperglycemia much faster than the HBMR animals that maintained initial blood sugar levels until the fifth week of the experiment (figure 1B). Furthermore, although STZ application resulted in a rapid development of hyperglycemia in all experimental groups, blood glucose levels were still highest in the LBMR line (figure 1B). Interestingly, the glucose profiles were strongly linked to body mass throughout the experiments, particularly in the L-BMR individuals (figure 1A). Thus, individuals with genetically determined low BMR have much less efficient glucose turnover and are therefore considerably more prone to develop obesity and hyperglycemic states than mice of the other two line types.

Studies on C57BL/6J mice fed HFD revealed that their fast weight gain is due to increased energy intake and linked to a reduced metabolic rate, as compared with the mice fed a normal diet. ${ }^{41}$ The nature of this reduction was nonetheless unidentified. Recent studies on our selected mouse line types demonstrated that HFD diet causes a significant drop in total spontaneous physical activity (SPA), and ultimately, energy expenditure, which may explain the metabolic reduction observed in Winzell and Ahrén's study. ${ }^{161}$ Nevertheless, irrespective of the energy density of a diet, HBMR mice manifested higher SPA (or conversely, LBMR mice manifested lower), which 
indicates the existence of a strong, positive link between BMR and SPA. ${ }^{16}$ This corresponds well with studies on humans showing that the nexus of low energy expenditures and sedentary lifestyle is associated with reduced activity of lipoprotein lipase, severe dyslipidemia, altered glucose uptake and, finally, IR. ${ }^{5344}$

\section{Metabolism-related lipid profile in IR and T2D groups}

Lipid profile abnormalities are the hallmark of development of obesity and IR. ${ }^{26}{ }^{44}$ When compared with controls, all animals studied showed an increasing lipid index under the HFD treatment, except for the level of TGs in H-BMR mice (figure 3). This may suggest that the HBMR animals have a superior ability to use fat, which delays the development of prodiabetic syndromes including hyperlipidemia and enhanced gluconeogenesis. The most likely involved intracellular pathways is that of the mTOR, which plays a fundamental role in de novo lipogenesis through the SREBPB1 dependent pathway in hepatocytes and white adipose tissue. ${ }^{24} 2631$ Recent studies on rat models show overexpression of SREBP1 gene in HFD-fed animals. ${ }^{31}$ Analogically, in our study, we found significantly greater activity of $m T O R$ in both high fat and STZ stimulated mice, particularly in the H-BMR line (figure 4). This suggests that triggered lipogenesis based on the mTOR-SREBPB1 signaling pathway may constitute a significant part of the molecular mechanism underlying genetically determined high BMR, which acts as a compensatory mechanism lowering propensity to the detrimental effects of western-type diet and sedentary lifestyle. ${ }^{121645}$ Conversely, a reduction in metabolic rate may stimulate weight gain, excess adiposity, and favor the development of IR and T2D.

\section{mTOR and glucose metabolism}

The results presented herein demonstrate BMR-related variation in the level of liver mTOR protein, with the HBMR mice manifesting higher mTOR expression than low BMR individuals (figure 4). Moreover, in both selected lines, we found a significant increase in the amount of $m$ TOR mRNA in HFD and T2D groups. As demonstrated in studies using mTOR inhibitors, the reduction of mTOR activity results in a deterioration in carbohydrate balance due to impaired insulin secretion, IR, or a combination of both. ${ }^{27}$ Inhibition of $m$ TOR is associated with an increased incidence of hyperglycemia and new-onset diabetes, ranging from $13 \%$ to $50 \%$ in clinical trials. ${ }^{276}$ However, an increase in activity of nutrient and insulin-sensing mTOR pathways stimulate glycolysis, glucose uptake, and glycogen synthesis while reducing protein breakdown and gluconeogenesis mostly in muscle and liver. ${ }^{24}{ }^{26}$ Moreover, the mTOR1 complex is a positive regulator of $\beta$-cell function, since its constitutive activation in pancreatic cells improves insulin secretion. ${ }^{227}$ It is therefore possible that a significantly higher food consumption rate of the HBMR animals promotes greater concentration of liver mTOR, which in turn, fosters a transition toward catabolic processes enabling the maintenance of high physical activity and increased level of general metabolism. ${ }^{16}{ }^{23}$ Such physiological adjustment may then act as a protection shield against disturbances in glucose homeostasis.

However, the effects of mTOR on glucose turnover remain complex, and many studies suggest that its role in promotion and development of IR and T2D is ambiguous. ${ }^{2428}$ Besides the role of mTOR in restoring patency of carbohydrates homeostasis, its chronic activation may also promote symptoms of IR. ${ }^{2}{ }^{27}$ For example, several studies showed that mTOR activity may be elevated in many organs of genetically obese or high-fat fed mice. ${ }^{24} 47$ Impairment of the PI3K-Akt pathway because of mTOR overactivity disrupts transport of insulin-regulated glucose transporter (GLUT4) to the cell membrane and therefore lowers the efficiency of glucose uptake. ${ }^{225} 27 \mathrm{It}$ seems that the relationship between mTOR activity and metabolic homeostasis follows a U-shaped curve, where too low or too high activity of mTOR has a deleterious effect on systemic metabolism. ${ }^{24}$

\section{Organismal metabolism as a risk factor for IR and T2D}

Although several studies indicate that above average energy intake may constitute a significant risk factor for many diseases (eg, cardiovascular diseases and cancer) or general multimorbidity, the relation between basal/ resting metabolism and propensity to weight gain and T2D development in humans is equivocal. ${ }^{78} 49$ However, the majority of studies report phenotypic effects, usually in obese patients often with already diagnosed diabetes of each type. ${ }^{14} 1839$ The observed associations between BMR, weight gain, and T2D may be a result of conditionrelated pathologies (eg, glycosuria and increased protein turnover) rather than genetically determined variation in energy expenditures. Moreover, Anthanont and Jensen ${ }^{50}$ analyzed a large database of volunteers participating in 17 years of monitoring and found that initial low BMR did not affect weight gain in a typical Western populations that were under study. Although Anthanont and Jensen's study suggests the lack of causative link between low BMR and subsequent weight gain, their results should be treated with caution, as all other correlative analyses lacking a control of genetic background. To bring progress to this field of study, the animal model presented may significantly contribute toward a better understanding of the causality between metabolic expenditures, body mass gain, and T2D.

\section{Conclusions}

There is still much to learn about the contribution of genetically conditioned metabolic rates to the susceptibility to develop metabolism-related diseases. In this study, we demonstrated that the HFD lines of mice divergently selected for BMR are a robust animal model for unraveling the associations between energy expenditures and T2D progression. Low energy expenditures of the L-BMR mice maintained at ad lib conditions and limited locomotory activity faithfully emulate the so-called 
sedentary lifestyle of the great majority of contemporary humans. H-BMR type mice defy this scenario as they maintain range-stable glucose homeostasis. Undoubtedly, incorporation of BMR-related approach in studies of metabolic diseases, including diabetes, should be of significant importance to indicate patients with predisposition to IR and T2D.

\section{Acknowledgements The authors are grateful to $\mathrm{N}$ Ochman for correcting the English.}

Contributors SM contributed to the design of the study, performed research, analyzed data, contributed to the discussion, wrote and edited the manuscript; DS and AS performed research and contributed to the discussion; SP, SB, MB, and GN performed research; MK and $\mathrm{HC}$ contributed to the design of the study, analyzed data, contributed to the discussion, and reviewed the manuscript. All authors approved the final version of the manuscript. SM is the guarantor of this work and, as such, had full access to all the data in the study and takes responsibility for the integrity of the data and the accuracy of the data analysis.

Funding This work was supported by Miniatura 1-DEC_2017/01/X/NZ4/00315 from the National Science Center, Poland.

Competing interests None declared.

Patient consent for publication Not required.

Ethics approval All procedures were approved by the Local Ethical Committee on Testing Animals in Olsztyn (approval 22/2017).

Provenance and peer review Not commissioned; externally peer reviewed.

Data availability statement Data are available on reasonable request.

Open access This is an open access article distributed in accordance with the Creative Commons Attribution Non Commercial (CC BY-NC 4.0) license, which permits others to distribute, remix, adapt, build upon this work non-commercially, and license their derivative works on different terms, provided the original work is properly cited, appropriate credit is given, any changes made indicated, and the use is non-commercial. See: http://creativecommons.org/licenses/by-nc/4.0/.

ORCID iD

Sebastian Maciak http://orcid.org/0000-0001-5652-8224

\section{REFERENCES}

1 Suman RK, Ray Mohanty I, Borde MK, et al. Development of an experimental model of diabetes co-existing with metabolic syndrome in rats. Adv Pharmacol Sci 2016;2016:9463476

2 Ardestani A, Lupse B, Kido Y, et al. Mtorc1 signaling: a doubleedged sword in diabetic $\beta$ cells. Cell Metab 2018;27:314-31.

3 Chao P-C, Li Y, Chang C-H, et al. Investigation of insulin resistance in the popularly used four rat models of type-2 diabetes. Biomed Pharmacother 2018;101:155-61.

4 Fabbri E, An Y, Schrack JA, et al. Energy metabolism and the burden of multimorbidity in older adults: results from the Baltimore longitudinal study of aging. J Gerontol A Biol Sci Med Sci 2015;70:1297-303.

5 Hamilton MT, Hamilton DG, Zderic TW. Role of low energy expenditure and sitting in obesity, metabolic syndrome, type 2 diabetes, and cardiovascular disease. Diabetes 2007;56:2655-67.

6 Atamni HJA-T, Mott R, Soller M, et al. High-Fat-Diet induced development of increased fasting glucose levels and impaired response to intraperitoneal glucose challenge in the collaborative cross mouse genetic reference population. BMC Genet 2016;17:1-19.

7 Piaggi P. Metabolic determinants of weight gain in humans. Obesity 2019:27:691-9.

8 Patron J, Serra-Cayuela A, Han B, et al. Assessing the performance of genome-wide association studies for predicting disease risk. PLoS One 2019;14:e0220215.

9 Feher J. Energy balance and regulation of food intake. Quantitative Human Physiology, 2012.

10 Bosy-Westphal A, Kahlhöfer J, Lagerpusch M, et al. Deep body composition phenotyping during weight cycling: relevance to metabolic efficiency and metabolic risk. Obes Rev 2015;16 Suppl 1:36-44. -

11 Caron N, Peyrot N, Caderby T, et al. Energy expenditure in people with diabetes mellitus: a review. Front Nutr 2016;3:56.
12 Kotz CM, Perez-Leighton CE, Teske JA, et al. Spontaneous physical activity defends against obesity. Curr Obes Rep 2017;6:362-70.

13 Bettini S, Favaretto F, Compagnin C, et al. Resting energy expenditure, insulin resistance and UCP1 expression in human subcutaneous and visceral adipose tissue of patients with obesity. Front Endocrinol 2019;10:548.

14 Buscemi S, Verga S, Caimi G, et al. Low relative resting metabolic rate and body weight gain in adult Caucasian Italians. Int $J$ Obes 2005;29:287-91.

15 Lazzer S, Bedogni G, Lafortuna CL, et al. Relationship between basal metabolic rate, gender, age, and body composition in 8,780 white obese subjects. Obesity 2010;18:71-8.

16 Sadowska J, Gębczyński AK, Konarzewski M. Metabolic risk factors in mice divergently selected for Bmr fed high fat and high CarB diets. PLoS One 2017:12:e0172892.

17 Luke A, Durazo-Arvizu R, Cao G, et al. Positive association between resting energy expenditure and weight gain in a lean adult population. Am J Clin Nutr 2006;83:1076-81.

18 Hebert SL, Nair KS. Protein and energy metabolism in type 1 diabetes. Clin Nutr 2010;29:13-17.

19 Katzmarzyk PT, Pérusse L, Tremblay A, et al. No association between resting metabolic rate or respiratory exchange ratio and subsequent changes in body mass and fatness: $5-1 / 2$ year follow-up of the Québec family study. Eur J Clin Nutr 2000;54:610-4.

20 Morino K, Kondo K, Tanaka S, et al. Total energy expenditure is comparable between patients with and without diabetes mellitus: clinical evaluation of energy requirements in patients with diabetes mellitus (CLEVER-DM) study. BMJ Open Diabetes Res Care 2019;7:e000648.

21 King AJF. The use of animal models in diabetes research. Br J Pharmacol 2012;166:877-94.

22 Al-awar A, Kupai K, Veszelka M, et al. Experimental diabetes mellitus in different animal models. J Diabetes Res 2016;2016:1-12.

23 Konarzewski M, Książek A. Determinants of intra-specific variation in basal metabolic rate. J Comp Physiol B 2013;183:27-41.

24 Laplante M, Sabatini DM. mTOR signaling in growth control and disease. Cell 2012;149:274-93.

25 Blagosklonny MV. TOR-centric view on insulin resistance and diabetic complications: perspective for endocrinologists and gerontologists. Cell Death Dis 2013;4:e964.

26 Mao Z, Zhang W. Role of mTOR in glucose and lipid metabolism. Int $J$ Mol Sci 2018;19:2043.

27 Vergès $\mathrm{B}$, Cariou B. mTOR inhibitors and diabetes. Diabetes Res Clin Pract 2015;110:101-8.

28 Vergès $B$, mTOR VB. mTOR and cardiovascular diseases: diabetes mellitus. Transplantation 2018;102:S47-9.

29 Tuo Y, Xiang M. mTOR: a double-edged sword for diabetes. $J$ Leukoc Biol 2019;106:385-95.

30 Gebczyński AK, Konarzewski M. Effects of oxygen availability on maximum aerobic performance in Mus musculus selected for basal metabolic rate or aerobic capacity. J Exp Biol 2011;214:1714-20.

31 Moreno-Fernández S, Garcés-Rimón M, Vera G, et al. High Fat/High glucose diet induces metabolic syndrome in an experimental rat model. Nutrients 2018;10:1502.

$32 \mathrm{Car} \mathrm{H}$, Żendzian-Piotrowska M, Prokopiuk S, et al. Ceramide profiles in the brain of rats with diabetes induced by streptozotocin. Febs $J$ 2012;279:1943-52.

33 Livak KJ, Schmittgen TD. Analysis of relative gene expression data using real-time quantitative PCR and the 2(-Delta Delta C(T)) Method. Methods 2001;25:402-8.

34 Marullo L, El-Sayed Moustafa JS, Prokopenko I. Insights into the genetic susceptibility to type 2 diabetes from genome-wide association studies of glycaemic traits. Curr Diab Rep 2014;14:551.

35 Loos RJ. The genetics of adiposity. Curr Opin Genet Dev 2018;50:86-95.

36 Stöckli J, Fisher-Wellman KH, Chaudhuri R, et al. Metabolomic analysis of insulin resistance across different mouse strains and diets. J Biol Chem 2017;292:19135-45.

37 Parilla JH, Willard JR, Barrow BM, et al. A mouse model of beta-cell dysfunction as seen in human type 2 diabetes. J Diabetes Res 2018;2018:6106051

38 Burnett CML, Grobe JL. Dietary effects on resting metabolic rate in C57BL/6 mice are differentially detected by indirect $(\mathrm{O} 2 / \mathrm{CO} 2$ respirometry) and direct calorimetry. Mol Metab 2014;3:460-4.

39 Poulsen P, Vaag A, Kyvik K, et al. Genetic versus environmental aetiology of the metabolic syndrome among male and female twins. Diabetologia 2001;44:537-43.

40 Willemsen G, Ward KJ, Bell CG, et al. The concordance and heritability of type 2 diabetes in 34,166 twin pairs from international twin registers: the discordant twin (DISCOTWIN) Consortium. Twin Res Hum Genet 2015;18:762-71. 
41 Winzell MS, Ahrén B. The high-fat diet-fed mouse: a model for studying mechanisms and treatment of impaired glucose tolerance and type 2 diabetes. Diabetes 2004;53 Suppl 3:S215-9.

42 Montgomery MK, Hallahan NL, Brown SH, et al. Mouse straindependent variation in obesity and glucose homeostasis in response to high-fat feeding. Diabetologia 2013;56:e1139:1129-39.

43 Wang $\mathrm{H}$, Eckel RH. Lipoprotein lipase: from gene to obesity. Am J Physiol Endocrinol Metab 2009;297:E271-88.

44 Huang Y, Li X, Wang M, et al. Lipoprotein lipase links vitamin $\mathrm{D}$, insulin resistance, and type 2 diabetes: a cross-sectional epidemiological study. Cardiovasc Diabetol 2013;12:17.

45 Kumareswaran K, Elleri D, Allen JM, et al. Physical activity energy expenditure and glucose control in pregnant women with type 1 diabetes: is 30 minutes of daily exercise enough? Diabetes Care 2013;36:1095-101.
46 Kleinert M, Sylow L, Fazakerley DJ, et al. Acute mTOR inhibition induces insulin resistance and alters substrate utilization in vivo. Mol Metab 2014;3:630-41.

47 Shigeyama Y, Kobayashi T, Kido Y, et al. Biphasic response of pancreatic beta-cell mass to ablation of tuberous sclerosis complex 2 in mice. Mol Cell Biol 2008;28:2971-9.

48 Maciak S, Michalak P. Cell size and cancer: a new solution to Peto's paradox? Evol Appl 2015;8:2-8.

49 Sawicka D, Maciak S, Kozłowska H, et al. Functional and structural changes in aorta of mice divergently selected for basal metabolic rate. J Comp Physiol B 2020;190:101-12.

50 Anthanont $\mathrm{P}$, Jensen MD. Does basal metabolic rate predict weight gain? Am J Clin Nutr 2016;104:959-63. 